\title{
Salvage chemotherapy with irinotecan, 5-fluorouracil and leucovorin for taxane- and cisplatin-refractory, metastatic gastric
}

\section{cancer}

\author{
ST Kim', WK Kang*,', JH Kang', KW Park', J Lee', S-H Lee', JO Park', K Kim', WS Kim', CW Jung', YS Park', \\ Y-H Im' and K Park'
}

'Division of Hematology and Oncology, Department of Medicine, Samsung Medical Center, Sungkyunkwan University School of Medicine, 50 Ilwon-dong, Kangnam-gu, Seoul, 135-710, Seoul, Korea

\begin{abstract}
We performed a phase II study of combination chemotherapy with irinotecan, 5-fluorouracil (5-FU) and leucovorin in metastatic gastric cancer patients who were previously treated with taxane and cisplatin, to evaluate the antitumour activity and toxicity of the combination chemotherapy. The metastatic gastric adenocarcinoma patients who were previously treated with taxane and cisplatin combination as first line, and had at least one measurable lesion, 0-2 ECOG performance status and adequate organ functions, were considered eligible. They received irinotecan $\left(150 \mathrm{mg} \mathrm{m}^{-2}\right.$, day I) and leucovorin $\left(100 \mathrm{mg} \mathrm{m}^{-2}\right.$, day I), followed by continuous infusion of $5-\mathrm{FU}\left(1000 \mathrm{mg} \mathrm{m}^{-2}\right.$ day $^{-1}$, days I and 2) every 2 weeks. Treatment was continued until progression of disease was observed. In all, 64 patients were treated with this combination chemotherapy. The median age of the patients was 55 years (range, 33-74 years), and the median ECOG performance status was I (0-I, 6I (95\%)). Out of 64 patients, 57 were assessable for response. Among 57 assessable patients, no complete response and 12 partial responses were observed (overall response rate, $21 \%$; 95\% confidence interval (Cl), 10-32\%). Stable disease was observed in 14 patients (25\%) and progressive disease in 31 patients (54\%). The median time to progression was 2.5 months $(95 \% \mathrm{Cl}, 1.6-3.4)$ and the median overall survival since the start of the second-line modified FOLFIRI was 7.6 months ( $95 \% \mathrm{Cl}, 6.5-8.7)$. Grade $3-4$ haematologic toxicities included neutropenia in seven patients ( 1 \%) and thrombocytopenia in five patients (8\%). Grade 3-4 nonhaematologic toxicities included diarrhoea in two patients (3\%) and vomiting in two patients (3\%). There were no treatment-related deaths. The combination of irinotecan, 5-FU and leucovorin showed moderate activity and favourable toxicity profile as a second-line treatment in metastatic gastric cancer patients, who were previously treated with taxane and cisplatin.
\end{abstract}

British Journal of Cancer (2005) 92, I850- |854. doi:I0.1038/sj.bjc.6602575 www.bjcancer.com

Published online 3 May 2005

(c) 2005 Cancer Research UK

Keywords: gastric carcinoma; irinotecan; 5-FU; salvage chemotherapy

Gastric adenocarcinoma is the most common malignancy and one of the major causes of cancer death in Korea (Bae et al, 2002). The prognosis of unresectable gastric cancer has been improved by palliative chemotherapy (Murad et al, 1993; Pyrhonen et al, 1995; Glimelius et al, 1997), but median overall survival (OS) rarely exceeds 1 year (Wils, 1998). Studies showed the benefit of combination regimens, such as fluorouracil (FU), doxorubicin and methotrexate (FAMTX) (Wils, 1998) or etoposide, leucovorin (LV) and FU (ELF), over best supportive care (Murad et al, 1993; Pyrhonen et al, 1995; Glimelius et al, 1997). Other combination regimens investigated include epirubicin, cisplatin and infusional FU (ECF) and 5-day infusional FU plus cisplatin (FUP). However, a randomised phase $\beta$ trial showed that there were no significant differences in response rates (RRs) or survival durations among the combination regimens. Although there are no standard first-

*Correspondence: Dr WK Kang; E-mail: wkkang @smc.samsung.co.kr Received 26 January 2005; revised 14 March 2005; accepted I4 March 2005; published online 3 May 2005 line chemotherapeutic drugs in unresectable gastric cancer, ECF is generally considered as a standard therapy in Europe after the results from phase III trial have been published (Webb et al, 1997).

Taxane and cisplatin has been used as a first-line chemotherapy with various schedules and doses since the late 1990s (Roth et al, 2000; Ridwelski et al, 2001; Kornek et al, 2002; Lee et al, 2004). Taxane and cisplatin chemotherapy has produced $30-50 \%$ of RRs as a first-line treatment, but the diseases of responding and nonresponding patients eventually progress (Roth et al, 2000; Ridwelski et al, 2001; Kornek et al, 2002; Lee et al, 2004). A randomised phase III trial showed a higher RR (39 vs 23\%) and longer time to progression (TTP; 5.2 vs 3.7 months) in DCF (docetaxel, cisplatin, 5-fluorouracil) arm when compared to $\mathrm{CF}$ (cisplatin, 5-fluorouracil) arm (Ajani et al, 2003).

In addition to novel combinations of established agents, the use of newer agents, such as irinotecan, is under investigation. Irinotecan, a topoisomerase I inhibitor, proved to have a clinical activity against unresectable gastric cancer. The RR of irinotecan alone has been reported to be $20-25 \%$ in phase II studies (Futatsuki et al, 1994; Kohne et al, 2003), regardless of prior 
chemotherapy. Combination of irinotecan with cisplatin showed the RR of $48 \%$ in metastatic gastric cancer patients who had received no or one chemotherapy regimen (Boku et al, 1999), and combination with 5-fluorouracil (5-FU) produced the RRs of $22-$ $42.4 \%$ in previously untreated gastric adenocarcinoma patients (Blanke et al, 2001; Assersohn et al, 2004; Bouche et al, 2004).

We performed a phase II study of a combination chemotherapy with irinotecan, leucovorin and 5-FU in metastatic gastric cancer patients, who were previously treated with taxane and cisplatin combination, to evaluate the antitumour activity and toxicity of the combination chemotherapy.

\section{MATERIALS AND METHODS}

\section{Eligibility}

Patients were eligible with histologically confirmed, unresectable adenocarcinoma of the stomach. They were required to be between 18 and 75 years, have at least one measurable lesion, Eastern Cooperative Oncology Group (ECOG) performance status $\leqslant 2$, and have a life expectancy at least 3 months. Eligible patients must have received one prior palliative chemotherapy with taxane and cisplatin. Adequate haematologic function (absolute neutrophil count (ANC) $\geqslant 1500 \mathrm{~mm}^{-3}$, platelet count $\geqslant 100000 \mathrm{~mm}^{-3}$ ), hepatic function (aspartate aminotransferase/alanine aminotransferase (AST/ALT) $\leqslant 3.0$ times the upper normal limit (UNL), bilirubin $\leqslant 1.25$ times the UNL), and renal function (serum creatinine $\leqslant 1.5$ times the UNL) were required. Prior history of another malignancy within 5 years of study entry, apart from basal cell carcinoma of the skin or carcinoma in situ of the uterine cervix, precluded participation in the current trial. Patients with concurrent uncontrolled medical illness were also excluded. All patients provided a written informed consent according to the guideline provided by the institutional review board.

\section{Chemotherapy}

Chemotherapy was administered through a central venous catheter (Hickman catheter or chemoport) placed in the subclavian vein, and ambulatory pumps were used for continuous infusion of 5-FU. Irinotecan $\left(150 \mathrm{mg} \mathrm{m}^{-2}\right.$, over $90 \mathrm{~min}$, day 1$)$, followed by leucovorin $\left(100 \mathrm{mg} \mathrm{m}^{-2}\right.$, over $2 \mathrm{~h}$, day 1$)$, and then followed by 5 -FU (1000 $\mathrm{mg} \mathrm{m}^{-2}$, over $24 \mathrm{~h}$, day 1 and 2 ) were given as an intravenous infusion. Cycles were repeated every 2 weeks. Before irinotecan infusion, $0.3 \mathrm{mg}$ atropine was subcutaneously administered to prevent cholinergic syndrome. A 5-hydroxytryptamine type 3 receptor antagonist was given as antiemetic prophylaxis immediately before chemotherapy and loperamide was provided for diarrhoea. Treatment was continued until documented disease progression, unacceptable toxicity, patients' refusal, or physicians' decision.

\section{Dose modification}

Chemotherapy was withheld if the ANC was $<1500 \mathrm{~mm}^{-3}$ or the platelet count was $<100000 \mathrm{~mm}^{-3}$ on day 1 . In this case, the complete blood cell count (CBC) was repeated at least weekly and chemotherapy was restarted as soon as the ANC reached $\geqslant 1500 \mathrm{~mm}^{-3}$ and platelet count $\geqslant 100000 \mathrm{~mm}^{-3}$. If nadir ANC was less than $500 \mathrm{~mm}^{-3}$, or the nadir platelet count was less than $50000 \mathrm{~mm}^{-3}$, the doses of irinotecan and 5-FU were reduced to 120 and $800 \mathrm{mg} \mathrm{m}^{-2}$, respectively. If grade $3-4$ nonhaematologic toxicity other than alopecia, the treatment was withheld until recovery to grade 0 or 1 and the doses of irinotecan and 5-FU were reduced to 120 and $800 \mathrm{mg} \mathrm{m}^{-2}$, respectively. If patients required a delay of longer than 2 weeks, they were treated off the protocol.

\section{Assessment of efficacy and toxicity}

At study entry, the following investigations were performed: full history taking and physical examination, $\mathrm{CBC}$, chemistry, chest $\mathrm{X}$ ray and computed tomography scan. History taking, physical examination, CBC and chemistry were repeated before each cycle. Computed tomography scans were performed to document the disease extent and to evaluate response to treatment, every three cycles and when needed for the confirmation of response and suspected disease progression. Responses were classified according to World Health Organization (WHO) criteria. Patients were considered assessable for response if they had early disease progression or had received at least three cycles of treatment with at least one tumour assessment. Response rate was calculated as the ratio of number of patients who achieved complete responses or partial responses to the number of assessable patients. Duration of response was calculated from the first day of treatment to the date on which progressive disease was first observed or of the last follow-up, for the group of responding patients. Time to progression was calculated from the first day of treatment to the date on which progressive disease was first observed or of the last follow-up. Overall survival was calculated from the first day of treatment to the date of death or last follow-up.

Toxicity was graded according to National Cancer Institute common toxicity criteria (NCI-CTC) version 2.0. (NCI-CTC Web site). The severity of any toxicities not defined in the NCI-CTC were graded as $1=$ mild, $2=$ moderate, $3=$ severe or $4=$ very severe.

\section{Statistical analysis}

Descriptive statistics were reported as proportions and medians. Kaplan-Meier estimates were used in the analysis of all time-toevent variables, and the 95\% confidence interval (CI) for the median time to event was computed. The dose intensity (DI) was calculated as the ratio of the total dose (expressed in milligrams) per square meter of the patient, divided by the total treatment duration expressed in days. In this calculation, the end of treatment was considered to be 14 days after day 1 of the last cycle of chemotherapy. The relative DI was calculated as the ratio of the DI actually delivered to the DI planned by the protocol.

According to Simon's two-stage optimal design, a sample size of 55 was required to accept the hypothesis that the true RR is greater than $25 \%$ with $90 \%$ power, and to reject the hypothesis that the RR is less than $10 \%$ with $5 \%$ significance. At the first stage, if there were fewer than four responses out of the initial 31 patients, the study would terminate. Although the target number of patients was 55 , we planned to recruit $20 \%$ more than the target number of patients considering dropout.

SPSS for Windows (SPSS Inc., Chicago, IL, USA) was used for statistical analysis.

\section{RESULTS}

\section{Patient characteristics}

From November 2001 to July 2003, 64 patients were enrolled. The clinical characteristics of enrolled patients are shown in Table 1. The median age of the patients was 55 years (range, 33-74 years) and the majority $(73 \%)$ of patients was male. The median ECOG performance status was one $(0-1,61(95 \%))$, and all patients had metastatic disease at study entry. Major involved organs were liver and intra-abdominal lymph nodes. Regimens of the prior chemotherapy are listed in Table 1, and all of theses patients showed progressive disease after taxane and cisplatin before entry into this study. 
Table I Patient characteristics

\begin{tabular}{lcr}
\hline Patient characteristics & No. of patients $(\mathbf{N}=\mathbf{6 4})$ & $\%$ \\
\hline Age, years & & \\
$\quad$ Median (range) & $55(33-74)$ & \\
Sex & & 27 \\
$\quad$ Female & 17 & 73 \\
Male & 47 & \\
ECOG status & & 5 \\
0 & 3 & 50 \\
I & 58 & 5 \\
2 & 3 & 14 \\
& & 50 \\
First-line regimen & & 36 \\
Paclitaxel/CDDP & 8 & \\
Docetaxel/CDDP & 33 & 41 \\
EDP & 23 & 7 \\
& & 6 \\
Organ involved & & 5 \\
Lymph nodes & 34 & \\
Liver & 29 & \\
Peritoneum & 6 & \\
Ovary & 5 & \\
Others & 4 & \\
\hline
\end{tabular}

Others: pancreas (two), bone (two), lung (one), adrenal (one), uterus (one), ureter (one). CDDP, cisplatin; EDP, epirubicin/docetaxel/cisplatin.

Table 2 Response rate

\section{Response}

Complete response

Partial response

Stable disease

Progressive disease

Total assessable patients
Number of patients (\%)

0

$12(21 \%)$

$14(25 \%)$

$31(54 \%)$

\section{Delivery of drugs}

The patients received a median of six (range, 1-9) cycles. The average relative dose-intensities were 0.87 for irinotecan, 0.87 for 5 -FU and 0.89 for leucovorin. Dose reduction was required in 11 patients ( 37 cycles) and treatment was delayed in 23 patients (59 cycles). The most common causes of dose reduction were neutropenia (64\%), thrombocytopenia $(45 \%)$, diarrhoea $(18 \%)$ and vomiting $(18 \%)$.

\section{Response rate}

In all, 57 patients were assessable for treatment response. Seven patients were excluded from the response analysis; four patients received one cycle and three patients two cycles of chemotherapy. Five of seven patients excluded from the analysis were lost to follow-up and the remaining two patients refused further treatment due to asthenia. Among 57 assessable patients, no complete response and 12 partial responses were observed (overall RR, 21\%; 95\% CI, 10-32\%). Stable disease was observed in 14 patients (25\%) and progressive disease in 31 patients (54\%) (Table 2). The median duration of response was 5.8 months (95\% CI, 4.0-7.6). The differences in RRs were not observed according to baseline patients' characteristics (age, sex, performance status, involved organs and previous chemotherapy regimen).

\section{Survival}

All 64 patients were in included in the survival analysis on an intent-to-treat basis. The median follow-up time was 22 months (95\% CI, 20-25). The median TTP was 2.5 months (95\% CI, $1.6-$ 3.4 ) and the median OS was 7.6 months (95\% CI, 6.5-8.7). The differences in TTP and OS were not observed according to age, sex, performance status, involved organs and previous chemotherapy regimen. The TTP and OS curves are shown in Figure 1.

\section{Toxicity}

Toxicities observed during the treatment are listed in Table 3. NCICTC grade 3 or 4 haematologic toxicities included neutropenia in
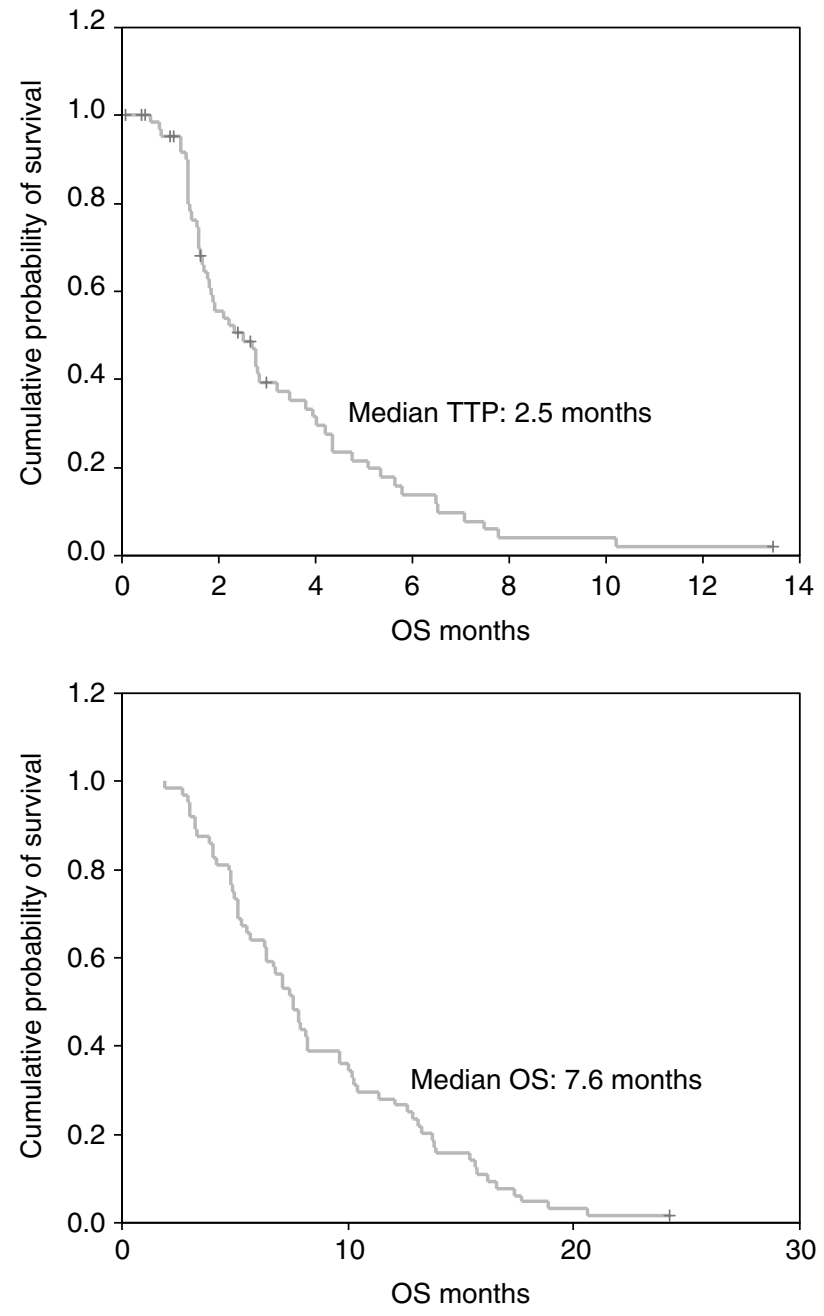

Figure I Time to progression and OS.

Table 3 Grade $3 / 4$ adverse events $(N=64)$

\begin{tabular}{lc}
\hline Toxicity & Number of patients (\%) \\
\hline Neutropenia & $7(11 \%)$ \\
Thrombocytopenia & $5(8 \%)$ \\
Diarrhoea & $2(3 \%)$ \\
Vomiting & $2(3 \%)$ \\
Total enrolled patients & 64 \\
\hline
\end{tabular}


seven patients (11\%) and thrombocytopenia in five patients (8\%). Two patients (3\%) with neutropenic fever and four patients $(6 \%)$ with grade 3 or 4 diarrhoea/vomiting required hospital admissions. There were no treatment-related deaths.

\section{DISCUSSION}

The prognosis of unresectable gastric cancer has remained poor despite new palliative treatment modalities. Response rates as a frontline of various combination chemotherapy regimens ranged between 30 and $70 \%$, but median OS rarely exceeded 10 months (Murad et al, 1993; Pyrhonen et al, 1995; Glimelius et al, 1997; Wils, 1998). Taxane and cisplatin combination chemotherapy regimen has been increasingly used as a first-line treatment in unresectable gastric cancer. Phase $\alpha$ studies with paclitaxel or docetaxel in combination with cisplatin with or without 5-FU have demonstrated RRs of $37-51 \%$ and OSs of 9-14 months in chemotherapy-naïve patients (Kollmannsberger et al, 2000; Ridwelski et al, 2001; Polee et al, 2002). Other phase II studies have employed the combination of irinotecan and cisplatin; RRs have ranged from 28 to $59 \%$ in chemotherapy-naïve patients, with corresponding OSs of 9-11 months (Shirao et al, 1997; Boku et al, 1999; Pozzo et al, 2001; Ajani et al, 2002). As most of the patients with unresectable gastric cancer eventually progress after the firstline chemotherapy, the second-line chemotherapy should be developed without cross-resistance to first-line chemotherapy. We studied a new second-line combination regimen, the modified FOLFIRI (irinotecan, leucovorin and 5-FU), in patients who had failed to taxane and cisplatin combination. We had used the reduced dose of irinotecan and 5FU as would be routinely used in similar regimens as in advanced colorectal cancer (i.e. the FOLFIRI regimen). The low dose of irinotecan $\left(150 \mathrm{mg} \mathrm{m}^{-2}\right.$, every 2 weeks) used in our study was based on the previous phase $\alpha$ studies conducted in Japan (Futatsuki et al, 1994; Bleiberg, 1999), which demonstrated modest antitumour activities in unresectable gastric cancer patients with irinotecan $150 \mathrm{mg} \mathrm{m}^{-2}$ every 2 -week schedule. This regimen showed a RR of $21 \%$ in assessable patients and of
$19 \%$ in all patients as a second-line treatment. In this phase II study, the median TTP was 2.5 months (95\% CI, 1.6-3.4 months) and the median OS was 7.6 months (95\% CI, 6.5-8.7 months). A previous study of irinotecan, 5-FU and leucovorin against gastric cancer yielded a RR of $22-40 \%$ and OS of 7.6-11.3 months as a first-line treatment (Blanke et al, 2001; Bouche et al, 2004). This three-drug combination regimens with different treatment scheme showed a RR of $29 \%$ and an OS of 6.4 months as a second-line treatment against oesophageal and gastric cancer (Assersohn et al, 2004).

The regimen showed a favourable toxicity profile. There were no treatment-related deaths. Although haematologic toxicity was reported, the incidence of grade 3-4 haematologic toxicity was approximately $10 \%$ (neutropenia in $11 \%$ and thrombocytopenia in $8 \%)$. NCI-CTC grades 3 or 4 nonhaematologic toxicities were minimal in incidence and all manageable (diarrhoea in $3 \%$ and vomiting in 3\%). Two previous studies using a similar regimen in gastric cancer showed $36 \%$ (Blanke et al, 2001) and $26 \%$ (Assersohn et al, 2004) of grade 3-4 neutropenia, and $28 \%$ (Blanke et al, 2001) and 8\% (Assersohn et al, 2004) of grade 3-4 diarrhoea. The significantly low rate of grade 3-4 toxicities in this study when compared to other irinotecan-based trials may be explained by the ethnic difference or the continuous infusion of 5-FU or the dose intensity of irinotecan. The ethnic difference in tolerability may be explained by the UDP-glucuronosyltranferase $1 \mathrm{~A} 1$ genotype, predictive factor of severe neutropenia (Innocenti et al, 2004). In addition, Blanke et al used 5-FU bolus, whereas we used continuous infusion of 5-FU. The dose intensities of irinotecan in the previous studies were $83 \mathrm{mg} \mathrm{m}^{-2}$ per week $\left(125 \mathrm{mg} \mathrm{m}^{-2}\right.$ per week in the first 4 weeks) (Blanke et al, 2001) and $90 \mathrm{mg} \mathrm{m}^{-2}$ per week (Assersohn et al, 2004), but the dose intensity in this study was $75 \mathrm{mg} \mathrm{m}^{-2}$ per week.

Based on a comparable activity and a favourable safety profile to other second-line regimens, the combination chemotherapy with irinotecan, 5-FU and leucovorin should be considered as a secondline treatment in taxane- and platinum-treated, unresectable gastric cancer.

\section{REFERENCES}

Ajani JA, Baker J, Pisters PW, Ho L, Mansfield PF, Feig BW, Charnsangavej C (2002) CPT-11 plus cisplatin in patients with advanced, untreated gastric or gastroesophageal junction carcinoma: results of a phase II study. Cancer 94: 641-646

Ajani JA, Cutsem E, Moiseyenko V, Tjulandin S, Fodor M, Majlis A, Boni C, Zuber E, Blattmann A (2003) Docetaxel, cisplatin, 5-fluorouracil compare to cisplatin (C) and 5-fluorouracil (F) for chemotherapy-naïve patients with metastatic or locally recurrent, unresectable gastric carcinoma (MGC): Interim results of a randomized phase $\beta$ trial (V325). Proc Am Soc Clin Oncol 22, (abstr. 999) p249

Assersohn L, Brown G, Cunningham D, Ward C, Oates J, Waters JS, Hill ME, Norman AR (2004) Phase II study of irinotecan and 5-fluorouracil/ leucovorin in patients with primary refractory or relapsed advanced oesophageal and gastric carcinoma. Ann Oncol 15: 64-69

Bae JM, Won WJ, Jung KW (2002) Annual report of the Korea Central Cancer Registry Program 2000: based on registered data from 131 hospitals. Cancer Res 34: 77-83

Blanke CD, Haller DG, Benson AB, Rothenberg ML, Berlin J, Mori M, Hsieh YC, Miller LL (2001) A phase II study of irinotecan with 5-fluorouracil and leucovorin in patients with previously untreated gastric adenocarcinoma. Ann Oncol 12: $1575-1580$

Bleiberg H (1999) CPT-11 in gastrointestinal cancer. Eur J Cancer 35: 371 379

Boku N, Ohtsu A, Shimada Y, Shirao K, Seki S, Saito H, Sakata Y, Hyodo I (1999) Phase II study of a combination of irinotecan and cisplatin against metastatic gastric cancer. J Clin Oncol 17: 319-323

Bouche O, Raoul JL, Bonnetain F, Giovannini M, Etienne PL, Lledo G, Arsene D, Paitel JF, Guerin-Meyer V, Mitry E, Buecher B, Kaminsky MC,
Seitz JF, Rougier P, Bedenne L, Milan C (2004) Randomized multicenter phase II trial of a biweekly regimen of fluorouracil and leucovorin (LV5FU2), LV5FU2 plus cisplatin, or LV5FU2 plus irinotecan in patients with previously untreated metastatic gastric cancer: a Federation Francophone de Cancerologie Digestive Group Study - FFCD 9803. J Clin Oncol 22: 4319-4328

Futatsuki K, Wakui A, Nakao I, Sakata Y, Kambe M, Shimada Y, Yoshino M, Taguchi T, Ogawa N (1994) Late phase II study of irinotecan hydrochloride (CPT-11) in advanced gastric cancer. CPT-11 Gastrointestinal Cancer Study Group. Jpn J Cancer Chemother 21: 1033-1038

Glimelius B, Ekstrom K, Hoffman K, Graf W, Sjoden PO, Haglund U, Svensson C, Enander LK, Linne T, Sellstrom H, Heuman R (1997) Randomized comparison between chemotherapy plus best supportive care with best supportive care in advanced gastric cancer. Ann Oncol 8: $163-168$

Innocenti $\mathrm{F}$, Undevia $\mathrm{SD}$, Iyer $\mathrm{L}$, Chen $\mathrm{PX}$, Das $\mathrm{S}$, Kocherginsky $\mathrm{M}$, Karrison T, Janisch L, Ramirez J, Rudin CM, Vokes EE, Ratain MJ (2004) Genetic variants in the UDP-glucuronosyltransferase 1A1 gene predict the risk of severe neutropenia of irinotecan. J Clin Oncol 22: $1382-1388$

Kohne CH, Catane R, Klein B, Ducreux M, Thuss-Patience P, Niederle N, Gips M, Preusser P, Knuth A, Clemens M, Bugat R, Figer I, Shani A, Fages B, Di Betta D, Jacques C, Wilke HJ (2003) Irinotecan is active in chemonaive patients with metastatic gastric cancer: a phase II multicentric trial. Br J Cancer 89: 997-1001

Kollmannsberger C, Quietzsch D, Haag C, Lingenfelser T, Schroeder M, Hartmann JT, Baronius W, Hempel V, Clemens M, Kanz L, Bokemeyer C (2000) A phase II study of paclitaxel, weekly, 24-h continous infusion 
5-fluorouracil, folinic acid and cisplatin in patients with advanced gastric cancer. Br J Cancer 83: 458-462

Kornek G.V, Raderer M, Schull B, Fiebiger W, Gedlicka C, Lenauer A, Depisch D, Schneeweiss B, Lang F, Scheithauer W (2002) Effective combination chemotherapy with paclitaxel and cisplatin with or without human granulocyte colony-stimulating factor and/or erythropoietin in patients with advanced gastric cancer. $\mathrm{Br} J$ Cancer 86: $1858-1863$

Lee SH, Kang WK, Park J, Kim HY, Kim JH, Lee SI, Park JO, Kim K, Jung CW, Park YS, Im YH, Lee MH, Park K (2004) Combination chemotherapy with epirubicin, docetaxel and cisplatin (EDP) in metastatic or recurrent, unresectable gastric cancer. $\mathrm{Br} J$ Cancer 91: $18-22$

Murad AM, Santiago FF, Petroianu A, Rocha PR, Rodrigues MA, Rausch M (1993) Modified therapy with 5-fluorouracil, doxorubicin, and methotrexate in advanced gastric cancer. Cancer 72: 37-41

NCI-CTC Web site. http://ctep.info.nih.gov

Polee MB, Eskens FA, van der Burg ME, Splinter TA, Siersema PD, Tilanus HW, Verweij J, Stoter G (2002) Phase II study of bi-weekly administration of paclitaxel and cisplatin in patients with advanced oesophageal cancer. Br I Cancer 86: 669-673

Pozzo C, Bugat R, Peschel C, Gorbunova V, Valvere V, Zaluski J, Biakhov M (2001) Irinotecan in combination with CDDP or 5-FU and folinic acid is active in patients with advanced gastric or gastro-oesophageal junction adenocarcinoma: final results of a randomised Phase II study. Proc Am Soc Clin Oncol 20, (abstr. 531) p134a
Pyrhonen S, Kuitunen T, Nyandoto P, Kouri M (1995) Randomised comparison of fluorouracil, epidoxorubicin and methotrexate (FEMTX) plus supportive care with supportive care alone in patients with nonresectable gastric cancer. Br J Cancer 71: 587-591

Ridwelski K, Gebauer T, Fahlke J, Kroning H, Kettner E, Meyer F, Eichelmann K, Lippert H (2001) Combination chemotherapy with docetaxel and cisplatin for locally advanced and metastatic gastric cancer. Ann Oncol 12: 47-51

Roth AD, Maibach R, Martinelli G, Fazio N, Aapro MS, Pagani O, Morant R, Borner MM, Herrmann R, Honegger H, Cavalli F, Alberto P, Castiglione M, Goldhirsch A (2000) Docetaxel (Taxotere)-cisplatin (TC): an effective drug combination in gastric carcinoma. Swiss Group for Clinical Cancer Research (SAKK), and the European Institute of Oncology (EIO). Ann Oncol 11: $301-306$

Shirao K, Shimada Y, Kondo H, Saito D, Yamao T, Ono H, Yokoyama T, Fukuda H, Oka M, Watanabe Y, Ohtsu A, Boku N, Fujii T, Oda Y, Muro K, Yoshida S (1997) Phase I-II study of irinotecan hydrochloride combined with cisplatin in patients with advanced gastric cancer. J Clin Oncol 15: $921-927$

Webb A, Cunningham D, Scarffe JH, Harper P, Norman A, Joffe JK, Hughes M, Mansi J, Findlay M, Hill A, Oates J, Nicolson M, Hickish T, O’Brien M, Iveson T, Watson M, Underhill C, Wardley A, Meehan M (1997) Randomized trial comparing epirubicin, cisplatin, and fluorouracil $v s$ fluorouracil, doxorubicin, and methotrexate in advanced esophagogastric cancer. J Clin Oncol 15: $261-267$

Wils J (1998) Treatment of gastric cancer. Curr Opin Oncol 10: 357-361 